Anaesthesist 2010 - 59:9-10 DOI 10.1007/s00101-009-1668-z

Online publiziert: 20. Januar 2010

(c) Springer-Verlag 2010

\author{
U. Kaisers ${ }^{1} \cdot$ R. Rossaint ${ }^{2}$ \\ ${ }^{1}$ Klinik und Poliklinik für Anästhesiologie und Intensivtherapie, \\ Universitätsklinikum Leipzig AöR \\ ${ }^{2}$ Klinik für Anästhesiologie, RWTH Aachen
}

\title{
Neue pandemische A(H1N1)-Influenza
}

\section{Sind unsere intensivmedizinischen Ressourcen ausreichend?}

Im April 2009 informierten die mexikanischen Gesundheitsbehörden über den Ausbruch eines neuen $\mathrm{H}_{1} \mathrm{~N}_{1}$-InfluenzaVirus in Mittelamerika, das sich rasch global ausbreitete [1]. Das Influenza-A-Virus gehört zur Gruppe der Orthomyxoviridae, die über ein breites Wirtsspektrum verfügen; ihr wichtigstes natürliches Reservoir stellen Wasservögel dar. Influenza-A-Viren sind behüllte Negativstrang-RNA-Viren, deren Hülle aus der Zellmembran gebildet wird und ganz überwiegend viruseigene Oberflächenproteine enthält. Besonders prominent sind Hämagglutinin (HA) und Neuraminidase (NA) sowie ein M2-Protonenkanal [2]. Influenza-A-Viren mit dem HA-Subtyp Hi traten vermutlich erstmals 1918 auf und lösten in der dem Ersten Weltkrieg folgenden schweren sozioökonomischen Krise eine kontinentübergreifende Pandemie mit geschätzt 20 Mio. bis 50 Mio. Todesfällen aus. Von besonderer Bedeutung ist die erhebliche genetische Variabilität der Influenza-A-Viren; bei einer Gesamtgröße des Genoms von rund 13.600 Nukleotiden wird eine Mutationsrate von $1-10 \%$ der Nachfolgeviren angenommen [2].

Das aktuelle Virus ist nach bisheriger Einschätzung zoonotischen Ursprungs und eine Reassortante aus einem nordamerikanischen H1-Stamm und dem eurasischen $\mathrm{H}_{1} \mathrm{~N}_{1}-\mathrm{Sch}$ weinegrippe-Virus. Dieses neue Influenzavirus zeigt eine ungewöhnliche Provinienz (Mittelamerika statt Asien), eine ungewöhnliche Aus- bruchssaison (Frühjahr statt Spätherbst) und neue Risikokollektive (Kinder, junge Erwachsene und Schwangere). Am 11.06.2009 erklärte die WHO die Pandemie mit dem „A( $\left.\mathrm{H} 1 \mathrm{~N}_{1}\right)$ swine-origin influenza virus" (S-OIV; [3]). Klinisch imponieren 3 intensivmedizinisch relevante Entitäten im Rahmen der neuen Influenza [4]:

- virusassoziierte Pneumonitis mit akutem Lungenversagen,

- sekundäre bakterielle Pneumonie und

- virusassoziierte Dekompensation einer „chronic obstructive pulmonary disease“ (COPD).

Aufgrund ihrer Lage auf der Südhalbkugel haben Australien und Neuseeland die diesjährige $\mathrm{H}_{1} \mathrm{~N}_{1}$-Influenza-Saison bereits hinter sich, sodass erste Erfahrungen zu Verlauf und Prognose der Erkrankung vorliegen. Insgesamt traten dort in den 3 Monaten von Juni bis August 2009 bei 25 Mio. Einwohnern 36.028 bestätigte Fälle von $\mathrm{H}_{1} \mathrm{~N}_{1}$ auf, von denen $13 \%$ entsprechend 18,6/100.0oo Einwohner zur Hospitalisierung führten [5]. In einer aktuellen Publikation wurde die Situation in allen 187 Intensivstationen (ITS) dieser Länder, die 722 Patienten mit bestätigter H1N1-Infektion behandelt hatten, evaluiert [6]. In dieser Studie betrug der Anteil adipöser Patienten $\left(\mathrm{BMI}>35 \mathrm{~kg} / \mathrm{m}^{2}\right)$ 29\%; Schwangere waren $\mathrm{zu} 9 \%$ betroffen, die Intensivletalitätsrate betrug 17\% (Variationsbreite 13,7-20,3\%). Zusätzlich liegen Daten der 15 australischen ITS vor, die auf die Behandlung des akuten Lungenversagens, einschließlich der Anwendung von extrakorporalem Gasaustausch mit

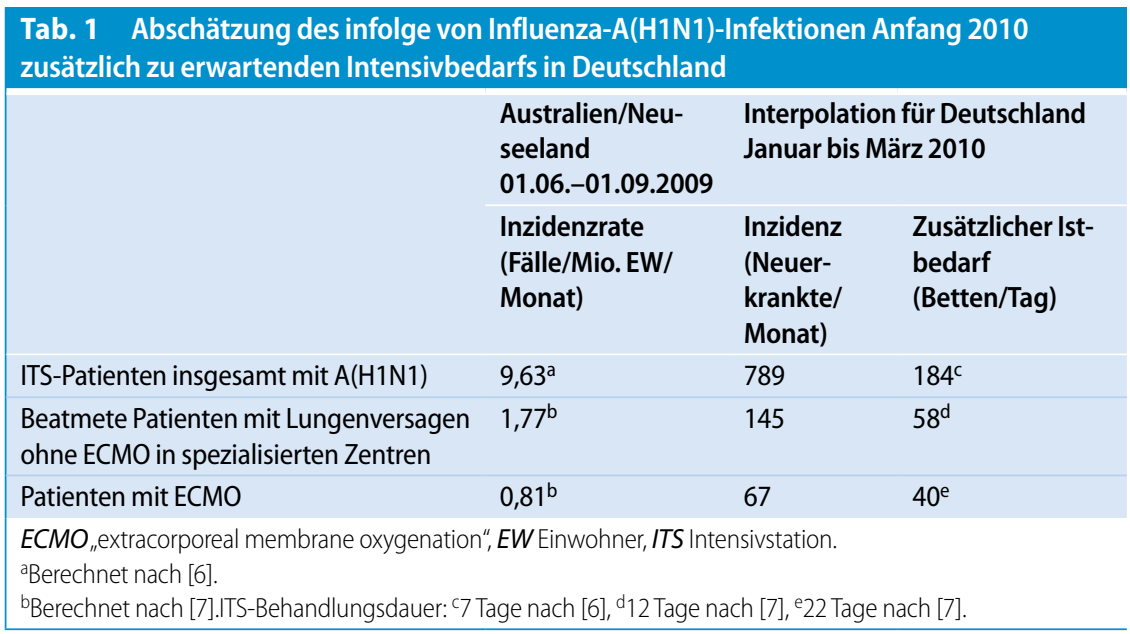


Membranlungen (ECMO), spezialisiert sind [7]. Der Anteil der dort behandelten Patienten mit akutem Lungenversagen und Nachweis des neuen $\mathrm{A}\left(\mathrm{H}_{1} \mathrm{~N}_{1}\right)$-Virus betrug während der dreimonatigen Influenzawelle 7,8 Fälle/1 Mio. Einwohner; 31\% dieser Patienten benötigten ECMO.

Daten aus Spanien bestätigten Schwangerschaft und Adipositas als Risikofaktoren für eine ITS-Aufnahme [8]. In einer Studie aus den USA, die den Nutzungsgrad von Intensivressourcen während der aktuellen $\mathrm{H}_{1} \mathrm{~N}_{1}$-Influenza berichtete, benötigten $25 \%$ der hospitalisierten Patienten eine Intensivtherapie; insgesamt $7 \%$ starben; dies entspricht einer ITS-Letalitätsrate von $28 \%$ [9]. Daraus ergibt sich auch für Deutschland die Frage, ob die Intensivtherapiemöglichkeiten für die neue $\mathrm{A}\left(\mathrm{H}_{1} \mathrm{~N}_{1}\right)$ Influenza ausreichend sein werden. Insbesondere die Untersuchungen aus Australien und Neuseeland $[6,7]$ sind wegen des vollständigen Einschlusses aller ITS hierbei von Bedeutung und ermöglichen eine Abschätzung der infolge $\mathrm{A}\left(\mathrm{H}_{1} \mathrm{~N}_{1}\right)$ benötigten Ressourcen, wie sie aktuell von Wenzel u. Edmond für die Vereinigten Staaten erfolgte [10]. Eine Extrapolation der australischen Daten auf Deutschland ist in - Tab. 1 dargestellt. Die hier überschlagene Kapazität muss additiv zu den ohnehin genutzten Intensivbetten mit ECMOOption für die Dauer von 2 Monaten verfügbar sein. Dabei ist zu berücksichtigen, dass die erforderlichen Intensivressourcen um noch einmal ca. 50\% höher anzusetzen wären, sofern der $\mathrm{H}_{1} \mathrm{~N}_{1}$-bedingte zusätzliche Bedarf nicht gleichmäßig während der Influenzasaison, sondern im Wesentlichen während 4 bis 6 Wochen auftreten sollte.

In der aktuellen Ausgabe von Der Anaesthesist entwickeln Bürkle et al. auf der Grundlage der derzeit vorliegenden Daten praktische Handlungsempfehlungen für die Intensivmedizin [11]. Die Zahlen zeigen, welche ernsthafte Herausforderung die zu erwartenden Influenza$\mathrm{A}\left(\mathrm{H}_{1} \mathrm{~N}_{1}\right)$-Infektionen bezüglich der Bereitstellung zusätzlicher Intensivressourcen darstellen könnte, die im Wesentlichen von den spezialisierten Einrichtungen mit ECMO-Option geleistet werden müsste. Bei Extrapolierung der australischen Daten wäre in Deutschland, selbst wenn die zusätzlichen Intensivkapazitäten zur Ver- fügung stünden, immer noch mit rund 100 Todesfällen nach Influenza-A(H1N1) bedingtem respiratorischen Versagen zu rechnen. Vor allem jüngere Patienten werden betroffen sein. Derzeit stehen in Deutschland ca. 50 Behandlungsplätze mit ECMO zur Verfügung (http://www. ardsnetwork.de/, 07.12.2009). Es erscheint kaum möglich, die entsprechenden Kapazitäten technisch und personell kurzfristig zu erhöhen. Dies unterstreicht die Bedeutung der in Deutschland angelaufenen Impfaktionen. Eine aktuelle Untersuchung der Centers for Disease Control (CDC) aus den USA weist darauf hin, dass bei $25 \%$ der gegen saisonale Influenza Geimpften im Alter von 18 bis 64 Jahren eine Kreuzreaktivität auch gegen das neue $\mathrm{A}\left(\mathrm{H}_{1} \mathrm{~N}_{1}\right)$-Virus vorlag [12]. Es ist daher postuliert worden, dass die Impfung gegen die saisonale Influenza ebenfalls zum Impfschutz gegen das neue $\mathrm{A}\left(\mathrm{H}_{1} \mathrm{~N}_{1}\right)-\mathrm{Vi}$ rus beiträgt [10]. So bleibt zu hoffen, dass beide Impfungen die Zahl der Patienten mit $\mathrm{H}_{1} \mathrm{~N}_{1}$ und schwerem respiratorischen Versagen auf ein beherrschbares Maß reduzieren werden.

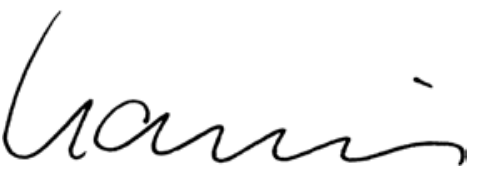

U. Kaisers

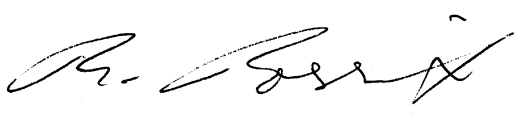

R. Rossaint

\section{Korrespondenzadresse}

Prof. Dr. U. Kaisers

Klinik und Poliklinik für Anästhesiologie und Intensivtherapie, Universitätsklinikum Leipzig AöR Liebigstraße 20, 04103 Leipzig

Udo.Kaisers@medizin.uni-leipzig.de

Interessenkonflikt. Der korrespondierende Autor gibt an, dass kein Interessenkonflikt besteht.

\section{Literatur}

1. Perez-Padilla $R$, de la Rosa-Zamboni $D$, Ponce de Leon S et al; INER Working Group on Influenza (2009) Pneumonia and respiratory failure from swine-origin influenza $A$ (H1N1) in Mexico. N Engl J Med 361:680-9.
2. Neumann G, Noda T, Kawaoka Y (2009) Emergency and pandemic potential of swine-origin $\mathrm{H} 1 \mathrm{~N} 1$ influenza virus. Nature 459:931-939

3. World Health Organization Regional Office for Europe (2009) WHO/Europe pandemic (H1N1) update, June 11 2009. http://www.euro.who.int/influenza/AH1N1/20090611_11

4. Moreno RP, Rhodes A, Chiche JD (2009) The ongoing $\mathrm{H} 1 \mathrm{~N} 1$ flu pandemic and the intensive care community: challenges, opportunities, and the duties of scientific societies and intensivists. Intensive Care Med 35:2005-2008

5. Australian Government Department of Health and Ageing (2009) Australian influenza surveillance summary report 2009, No.18, pp 1-22, http:// www.health.gov.au/internet/main/publishing.nsf/ Content/cda-surveil-ozflu-flucurr.htm/\$FILE/ozfluno18-2009.pdf

6. ANZIC Influenza Investigators, Webb SA, Pettilä V, Seppelt l et al (2009) Critical care services and 2009 H1N1 influenza in Australia and New Zealand. N Engl J Med 361:1925-1934

7. Australia and New Zealand Extracorporeal Membrane Oxygenation (ANZ ECMO) Influenza Investigators, Davies A, Jones D, Bailey M et al (2009) Extracorporeal membrane oxygenation for 2009 influenza $A(H 1 N 1)$ acute respiratory distress syndrome. JAMA 302:1888-1895

8. Rello J, Rodríguez A, Ibañez P et al; the H1N1 SEMICYUC Working Group (2009) Intensive care adult patients with severe respiratory failure caused by influenza A(H1N1)v in Spain. Crit Care 13:R148

9. Jain S, Kamimoto L, Bramley AM et al; Pandemic Influenza A (H1N1) Virus Hospitalizations Investigation Team (2009) Hospitalized patients with 2009 H1N1 influenza in the United States, April June 2009. N Engl J Med 361:1935-1944

10. Wenzel RP, Edmond MB (2009) Preparing for 2009 H1N1 influenza. N Engl J Med 361:1191-1193

11. Bürkle MA, Frey L, Zwißler B (2010) Die neue Influenza a H1N1/09 Virus Pandemie - eine Herausforderung für die Intensivmedizin. Anaesthesist 59:11-22

12. Centers for Disease Control and Prevention (CDC) (2009) Serum cross-reactive antibody response to a novel influenza $A(\mathrm{H} 1 \mathrm{~N} 1)$ virus after vaccination with seasonal influenza vaccine. MMWR Morb Mortal Wkly Rep 58(19):521-524 